\title{
Prevention of spinal cord injuries caused by diving: evaluation of the distribution and usage of a diving safety video in high schools
}

\author{
Vinay M Bhide, Virginia E Edmonds, Charles H Tator
}

\begin{abstract}
Objective-To determine and assess the distribution and use of Sudden Impact, a video designed by Think First and SportsSmart Canada, to help prevent spinal cord injury caused by careless shallow water diving among teenagers in the high risk group (15-24 years old).

Design-Survey of 92 public secondary schools in Toronto, Canada.

Subjects-The heads of the physical and health education departments of the 92 secondary public schools in the Metropolitan Toronto region.

Results-The response rate was $64 \%$ (59 schools), of which $76 \%$ (45) had actually received the video. Forty one schools $(91 \%)$ of those that received the video reported using it. Eighty per cent of responding schools showed it to grade 11 students. Eighty per cent of schools with swimming pools used the video compared with only $42 \%$ of schools without swimming pools.

Conclusions-There is a need for improvements in the system of distribution to ensure greater use of material such as this video. These may include direct distribution to principals, continuing contact with the schools, or mandatory inclusion of diving safety into the school curriculum.

(Injury Prevention 2000;6:154-156)
\end{abstract}

Keywords: diving; spinal cord injury; video; education

Spinal cord injury is a major public health problem and a leading cause of disability. ${ }^{1}$ Sports and recreation related spinal cord injury represents $10 \%-30 \%$ of all such injuries in various countries. ${ }^{2}$ In Ontario, Canada from 1948-83, diving accounted for $58.9 \%$ of all recreational related spinal cord injury. ${ }^{3}$ This amounts to approximately 60 major spinal injuries yearly caused by diving in this province alone. ${ }^{4}$ Although few of these injuries are fatal, many cause major disability because in almost all cases the cervical cord is involved. ${ }^{56}$ Ninety per cent of cord injuries due to diving result in complete or incomplete quadriplegia, whereas only $50 \%$ of spinal cord injury of all causes result in some form of quadriplegia. ${ }^{7}$

The typical victims of spinal cord injury caused by aquatic activities are teenage males in unsupervised recreational activities in the summer months. Diving is the most frequent type of aquatic activity leading to spinal cord injury $^{8}$ and almost $50 \%$ of these injuries involve persons between 15 and $24 .{ }^{9}$ Most victims survive but incur lifelong disability and immense financial costs.
Methods

THE VIDEO

Because we believed most of these injuries were preventable, in 1992, SportSmart Canada, the research division of Think First Canada-Penser d'Abord, a national brain and spinal cord injury prevention program, produced a video. It was entitled Sudden Impact and designed to alert teenagers about diving related spinal cord injury. The focus is on the dangers of shallow water diving and other careless behaviours associated with both supervised and unsupervised aquatic settings. The 20 minute video chronicles the histories of seven survivors of spinal cord injury all between 15 and 24, who are now all quadriplegic as a result of a diving injury. The video displays alarming statistics about shallow water diving and aquatic injury to illustrate the need for their prevention. Accompanying the video is a Leader's Guide, containing supplementary information, to be used by the classroom teacher as a tool for further classroom discussion.

\section{EVALUATION}

Although other evaluations of the Think First head injury and spinal cord injury prevention program have been conducted, ${ }^{10}{ }^{11}$ there has not been an objective assessment of a diving safety promotion strategy, such as Sudden Impact. Accordingly, the purpose of the present study was to assess the use and awareness of the video in public secondary schools - that is, a process evaluation and not one addressing behavioural change.

\section{MEASURES}

A questionnaire was sent to the heads of the physical and health education departments of all 92 public secondary schools in the six boards of education in the Metropolitan Toronto region. The questionnaire asked about the frequency of use of the video in the 1994-95 school year; its accessibility; the number of students who saw it, their grades; and how and where the video was used. Other questions addressed use of the Leader's Guide. Respondents were encouraged to supply any additional feedback.

All the public schools boards received the video but private or separate school boards were excluded from the study. The questionnaire was sent by facsimile to the appropriate person in each school. Confirmation of its receipt was obtained through follow up telephone calls and facsimile reports. Each respondent received up to three follow up phone calls if the questionnaire was not returned.
Street, Toronto, Ontario

M5T 2S8, Canada (e-mail:

charles.tator@uhn.on.ca) 


\section{Results}

The response rate was $64 \%$ (59 schools) of whom $80 \%$ (47) were aware of the Sudden Impact video, and of these, $76 \%$ reported that their school had received it. It is not evident why it failed to reach the remaining $24 \%$ (14). Ninety one per cent (41) of schools that had the video used it during the school year while the remainder stated that they planned to show it in the following year. Eighty per cent of schools with swimming pools showed the video compared with $42 \%$ of schools without swimming pools.

Sudden Impact was shown a mean of six times per school (range 1-39) to a mean of 174 students per school (22-975). In all, 6793 saw the video, however, the mean per cent of students in each school who saw it was only $16 \%(1 \%-$ $95 \%)$. The video was shown to high school grades 9-13 (ages 14-18), although the per cent of each grade who saw it ranged from $29 \%$ to $80 \%$. Only $46 \%$ of schools used the accompanying Leader's Guide to facilitate classroom discussion and for teacher preparation.

The "additional feedback" section of the survey contained no negative comments but some constructive criticisms. Thirty five respondents stated that the directness of the video strongly reinforced its message, and that students were very moved. Many students told their teachers that they would be more careful in the future and that their previous view that this type of injury could not happen to them was dispelled by watching the video. The video also stimulated class discussion about the causes, nature, and severity of these injuries. Many teachers expressed the opinion that viewing the video should be mandatory. The most frequent criticism was that the video featured no members of ethnoracial minorities.

\section{Discussion}

In the field of aquatic spinal injuries, several major prevention efforts have been launched in various countries in recent years. They include media campaigns, often involving celebrities, educational programs in high schools, changes in regulations concerning permissible depth for diving and other features of swimming pool design, and improved standards of spinal injury rescue and immobilization techniques. ${ }^{7}$ Nevertheless, many authors continue to reiterate the need for further prevention efforts. ${ }^{12-14}$

The timing of prevention strategies is considered crucial so that the messages reach the target population before the diving season begins. ${ }^{4} 1516$

The survey showed that almost all schools that received Sudden Impact used it. This suggests that this is an effective way of providing prevention materials to schools. However, almost one quarter of the surveyed schools did not have a copy of the video even though the distribution strategy was designed to deliver one to every public high school in Ontario. Possible reasons for the distribution failure include personnel changes in the school or school boards, lack of communication between the boys and girls physical and health education personnel within a school, the video reaching the wrong person, or being misplaced.
The response rate of $64 \%$ prompts consideration about non-respondents. Some may not have replied because the survey was conducted at a busy time in the academic schedule. It is also likely that some non-respondents did not have the video, or were unaware of it, and therefore, did not participate. Clearly, there is a need to improve the method of distribution of this and similar educational or motivational material.

It was interesting to note that the percentage of teenagers who saw the video at schools with a swimming pool was nearly double the percentage who saw it in schools without a pool. This result is not unexpected as schools with pools probably feel the need to emphasize water safety more than the others. However, the students in the latter schools are no less susceptible to spinal cord injury and must be reached equally. The only way to ensure this is by incorporating Sudden Impact into the physical and health education curriculum as mandatory viewing by all students.

A disappointing result was that only $16 \%$ of the students in each school saw the video. A likely explanation is that the video was shown most frequently in physical and health education classes and students enrolled in these courses only comprise between $15 \%-20 \%$ of the population in the schools surveyed (physical and health education is not compulsory after grade 10). These findings suggest that future distribution of prevention materials should not be restricted to physical and health education departments. A better strategy would be to send them to the headmaster or principal to ensure viewing by most studentsfor example, in assemblies.

\section{Implications for prevention}

The school setting represents an ideal opportunity to teach injury prevention and reach large numbers of students of diverse social and cultural backgrounds in an inexpensive fashion. The results suggest, however, that the distribution mechanism requires major improvements as described above. Some method to ascertain receipt of the material such as a return postcard is essential. Continuing contact with the schools is likely to be necessary to encourage greater use of the material. Showing a video to assemblies especially as part of a compulsory curriculum on injury prevention would ensure greater use. These measures to improve the distribution and use of this prevention program should also apply to other school based injury programs. In all cases, however, parallel efforts are needed to ensure that the educational material succeeds in changing behaviour in the desired manner.

This research was supported by the Easter Seals Research InstiThis research was supported by the Easter Seals Research Insti-
tute Summer Studentship numbers 9502 and 9604 . Grateful acknowledgment is made to Unilever Canada and its subsidiary Dickie Dee Ice Cream and to Sun Life Assurance Company of Canada for corporate sponsorship and to the Ontario Ministry of Culture, Tourism, and Recreation for government sponsorof Culture, Tourism,
ship of this program.

1 Cushman R. Injury prevention: the time has come. Can Med Assoc 7 1995;152:21-3.

2 Tator CH, Duncan EG, Edmonds VE, et al. Changes in epidemiology of acute spinal cord injury from 1947 to 1981 . Surg Neurol 1993;40:207-15. 
3 Tator $\mathrm{CH}$, Edmonds VE. Sports and recreation are a rising cause of spinal cord injury. Physician and Sportsmedicine 1986;14:157-67.

4 Tator CH, Palm J. The issue of spinal injuries due to diving and aquatic activities. Royal Life Saving Society of Canada Proceedings of the Symposium: Aquatic Spinal Injuries. Toronto: Royal Life Saving Society of Canada, 1980:9-11.

5 Bailes JE, Herman JM, Quigley MR, et al. Diving injuries of the cervical spine. Surg Neurol 1990;34:155-8.

6 Kluger Y, Jarosz D, Paul DB, et al. Diving injuries: a preventable catastrophe. F Trauma 1994;36:349-51.

7 Samples P. Spinal cord injuries: the high cost of careless diving. Physician and Sportsmedicine 1989;17:143-8.

8 Tator CH, Edmonds VE, New ML. Diving may be a breakneck affair. Royal Life Saving Society of Canada Ontario Branch Technical fournal 1979;1:5-14.

9 McQueen DM, Tenn L. Working towards spinal cord injury prevention in adolescents. L'Axone September 1990: 24-7.

10 Wright M, Rivara FP, Ferse D. Evaluation of the Think First head and spinal cord injury prevention program. Inj Prev 1995;1:81-5.

11 Avolio AEC, Ramsey FL, Neuwelt EA. Evaluation of a program to prevent head and spinal cord injuries: a comparison between middle school and high school. Neurosurgery 1992;31:557-61.

12 Grundy D, Penny P, Graham L. Diving into the unknown. BMF 1991;302:670-1.

13 DeMers G. To dive or not to dive: what depth is safe? Fournal of Physical Education, Recreation and Dance April 1994: $17-22$.

14 Blanksby BA, Wearne FK, Elliott BC, et al. Aetiology and occurrence of diving injuries. A review of diving safety. Sports Med 1997;23:228-46.

15 Tator CH, Edmonds VE. Diving: a frequent and potentially preventable cause of spinal cord injury. Can Med Assoc $\mathcal{F}$ 1981;124:1323-4.

16 DeVivo MJ, Sekar P. Prevention of spinal cord injuries that occur in swimming pools. Spinal Cord 1997;35:509-15.

\section{Appendix 1}

SUDDEN IMPACT QUESTIONNAIRE (YES/NO RESPONES)

1. Does your school have a swimming pool and/or other aquatic facility?

2. Are you aware of the public education video for the prevention of spinal cord injuries, Sudden Impact?

3. If yes, has your school/department received a copy of the video within the last two years?

4. If no, was the video at least made accessible to your department/school?

5. If the video was available to you, was it used?

If no, please answer question 6.

If yes, please answer questions 7-14.

6. Why was the video not used? (that is, haven't received a copy, using another video, etc)

7. How many times was the video used in the 1994-95 school year?

8. How many students saw the video?

9. What percentage of students in your school saw the video during the 1994-95 school year?

10. What class(es) was the video shown in? (that is, health, physical education, other, etc)

11. What grade levels was it being used with?

12. Was the accompanying resource guide used to supplement the video?

13. If yes, in what way? (that is, class discussion, assigned reading, etc)

14. Please provide us with any feedback on the video and/or accompanying resource, that is, was it helpful? Did it stimulate discussion? Etc

\section{Babysling related injuries: a case report and literature review}

\author{
J Brooks, F Finlay
}

\section{Case report}

A 17 day old baby slipped from her babysling onto the pavement hitting the front of her head, when her mother accidentally tripped and fell over. The baby cried immediately, but her mother brought her to the accident and emergency department, as she was concerned that she had bumped her head.

On examination two large parietal swellings were noted, and skull radiography confirmed bilateral fractures extending from the coronal sutures anteriorly, to the lamboidal sutures posteriorly. The child was well, and as neurological assessment was normal no further investigations were done. The baby was observed on a paediatric ward and had hourly observations overnight - pulse rate, blood pressure, pupil diameter, and response to light. These all remained within normal limits.

The next morning the child was described by her mother as "irritable" and she attributed this to the fact that her child had been wakened hourly. General examination was unremarkable apart from the parietal swellings previously noted; she was therefore allowed to sleep undisturbed for several hours. On waking, she appeared well and she was therefore allowed home. Her parents were told that they could return with her to the ward at any time, if they had concerns.
She returned three days later as her parents were worried about increasing drowsiness. On assessment she was awake and alert, and neurological examination revealed no abnormality. However in view of her history computed tomography of her brain was arranged. This showed a small right sided subdural haemorrhage with no midline shift. She was once again allowed home, and follow up one week later revealed a well child, with resolving parietal swellings.

\section{Discussion}

Babyslings are commonly used to carry young babies by strapping the child to the front of an adult's torso (see fig 1). Such slings may be used from birth (weight $3.5 \mathrm{~kg}$ ) to approximately 12 months of age $(12 \mathrm{~kg})$. After this age, a back carrier is recommended to carry heavier children.

Data from one UK marketing company Fickett and Stevens Associates (5 Hillgate St, London W8 7SP) shows that sales of babyslings are on the increase. Total sales of slings/ back carriers were up by $10 \%$ in April 1999 compared with December 1997. Approximately 300000 slings/carriers were sold in 1998, that is one sling per three children born in the UK. This figure does not include secondhand sales figures. Clearly, this method of carrying children is popular. 


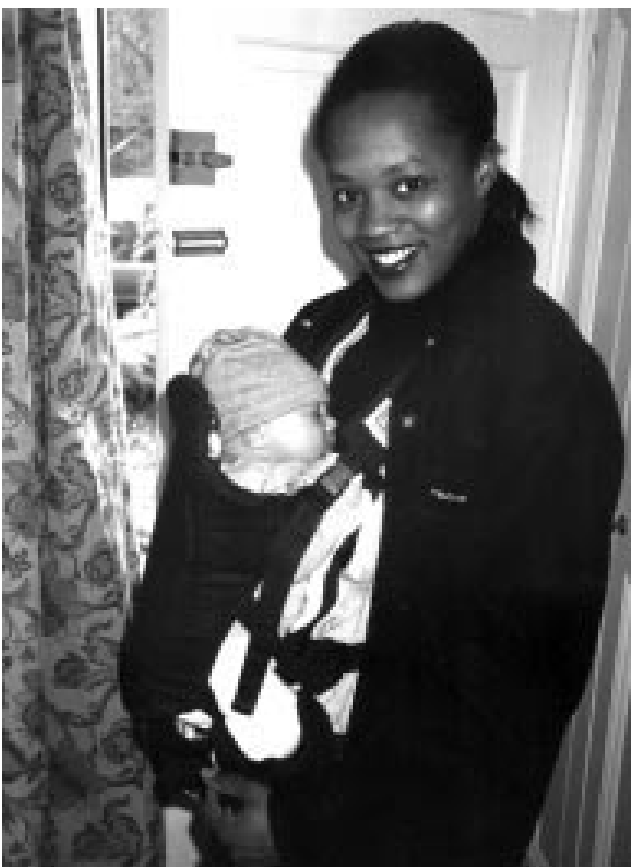

Figure 1 Babysling (photograph reproduced with permission).

A survey done by the Department of Trade and Industry from 1992-97 in 18 hospitals in the UK showed that there were 20 reported cases of sling related injuries. There were no fatal injuries, but two babies were admitted, one of whom had a skull fracture. ${ }^{1}$

Injury data obtained from the database of the Canadian Hospitals Injury Reporting and Prevention Program (CHIRPP) showed that there were 105 reports of sling related injuries seen in the emergency rooms of 10 paediatric and six general hospitals. ${ }^{2}$ Data collection from these hospitals began in 1990 in the paediatric hospitals and between 1991 and 1995 in the general hospitals. Nearly half of the injuries $(44.8 \%)$ were seen in babies under 6 months, and one third of the injuries happened at home, and another $18.1 \%$ of the injuries occurred on roads, sidewalks, or in parking areas. In $35.2 \%$ the child was injured when the person carrying them tripped or fell. In $19 \%$ the baby had fallen out of the carrier while being carried, in another $19 \%$ injuries to babies occurred while the child was in the carrier but it was not being worn. In $5.7 \%$ injury occurred to the child while the carrier was being put on or taken off, or the child was being put in or taken out of the carrier. The majority of injuries were to the head or face $(61 \%)$, and $10 \%$ of children had a skull fracture. Altogether $9.6 \%$ required hospital admission, 30\% required treatment but were well enough to go home, and 51\% were discharged with advice only.

Data on injuries from baby carriers have not been obtained for the US. However, a recent report has indicated that over 300000 front and back baby carriers have been recalled in the US because small infants shifted to one side and slipped through a leg opening, and fell to the ground. Thirteen such babies were reported to the Consumer Product Safety Commission. Babies most at risk were less than 2 months old. One of these babies suffered a fractured skull, and a further two infants received bruises. ${ }^{3}$

Neurological observation of young babies is difficult to undertake and interpret. The Glasgow coma scale is inappropriate for use in young children, as normal verbal and motor responses embodied in the standard Glasgow coma scale are not achievable in the first few years of life. Other scales have been devised which takes into account a child's age and therefore neurological immaturity, however additional information such as assessment of behaviour according to the main care giver needs to be considered. ${ }^{4}$

Guidelines for computed tomography of head injured children ${ }^{6}$ suggest that it is necessary in a child who has a fracture and a Glasgow coma score of less than 15 , which is the maximum score. However the coma scale may be difficult to interpret. Intracranial injury may occur with few or subtle signs and symptoms, if any, especially in babies less than 12 months old. ${ }^{7}$ The absence of a skull fracture does not exclude intracranial injury. ${ }^{8}$ Other studies have demonstrated a poor correlation between clinical symptoms and findings on computed tomography. ${ }^{9}$

There should therefore be a low threshold for cranial imaging of young infants with head injuries, as clinical assessment may be very difficult.

\section{Conclusion}

A Medline search using headings of "babysling", "harness" and "popousse", failed to reveal any studies on the safety of babysling or further information on sling related injuries. However, from our data, it appears that babysling related injuries are uncommon both in the UK and Canada.

The authors recommend that simple safety measures are taken such as ensuring that the child fits snugly in the harness, with the head supported if the baby is less than 6 months, and body straps and limb restraints to hold the baby in position. The person carrying the young infant should use an arm to keep the child close to their torso.

1 Department of Trade and Industry. Babyslings and backpack injuries. London: DTI, 1992-97.

2 Child Injury Division, Bureau Of Reproductive and Child Health, Laboratory Centre For Disease Control, Canada. CHIRPP database. Ottawa: Child Injury Division, Bureau Of Reproductive and Child Health, Laboratory Centre For Disease Control, Canada, 1999.

3 Anonymous. News and notes. Falls from soft infant carriers. Inj Prev 1999;5:257.

4 Simpson DA Cockington RA, Hanieh A, et al. Head injuries in infants and young children: the value of the paediatric coma scale. Review of literature and report on a study. Child's Nerv Syst 1997;7:283-90.

5 Westbrook A. The use of a paediatric coma scale for monitoring infants and young children with head injuries. Nursing in Critical Care 1997;2:72-5.

6 Advanced Life Support Group. Advanced paediatric life support. 2nd Ed. London: BMJ Publishing Group, 1997.

7 Murshid WR. Management of minor head injuries: admission criteria, radiological observation, and treatment of complications. Acta Neurochir (Wien) 1998;140:56-64.

8 Lloyd DA, Carty H, Patterson M, et al. Predictive value of skull radiography for intracranial injury in children with
blunt head injury. Lancet 1997;349:821-4.

9 Dietrich AM, Bowman MJ, Ginn-Pease ME, et al. PaediatDietrich AM, Bowman MJ, Ginn-Pease ME, et al. Paediat-
ric head injuries: can clinical factors reliably predict an ric head injuries: can clinical factors reliably predict an
abnormality on computed tomography. Ann Emerg Med 1993;22:1535-40. 